\title{
Atenção nutricional no pré-natal e no puerpério: percepção dos gestores da Atenção Básica à Saúde
}

\author{
The nutritional care during the prenatal and \\ postpartum: Perceptions of managers \\ of Primary Health Care
}

Anna Sylvia de Campos LAPORTE-PINFILDI ${ }^{1}$

Lia Thieme Oikawa ZANGIROLANI ${ }^{2}$

Natália SPINA ${ }^{3}$

Paula Andrea MARTINS 4

Maria Angélica Tavares de MEDEIROS²

\section{R E S U M O}

\section{Objetivo}

Avaliar a percepção dos gestores quanto à atenção nutricional ao pré-natal e ao puerpério na rede básica de município da Baixada Santista, São Paulo, segundo estrutura e processo.

\section{Métodos}

Realizou-se censo das 28 unidades básicas da área insular por meio de entrevistas com gestores. Avaliou-se a atenção nutricional segundo infraestrutura, processo de trabalho e vigilância alimentar e nutricional.

\section{Resultados}

A inserção do nutricionista foi o principal componente insuficiente da dimensão estrutura, interferindo negativamente na atenção nutricional para $60 \%$ dos gestores. Encontrou-se baixa conformidade para as seguintes

1 Universidade Federal de São Paulo, Instituto Saúde e Sociedade, Programa de Pós-Graduação em Ensino em Ciências da Saúde. Santos, SP, Brasil.

2 Universidade Federal de São Paulo, Instituto Saúde e Sociedade, Departamento de Políticas Públicas e Saúde Coletiva. Campus Baixada Santista, R. Silva Jardim, 136, Vila Mathias, 11015-020, Santos, São Paulo, Brasil. Correspondência para/ Correspondence to: MAT MEDEIROS. E-mails: <angelica.medeiros@unifesp.br>; <angelicamedeiros@gmail.com>.

${ }^{3}$ Universidade Federal de São Paulo, Escola Paulista de Medicina, Programa de Pós-Graduação em Nutrição. São Paulo, SP, Brasil.

4 Universidade Federal de São Paulo, Instituto Saúde e Sociedade, Departamento de Ciências do Movimento Humano. Santos, SP, Brasil.

Artigo baseado na dissertação de ASC LAPORTE-PINFILDI, intitulada "A estratégia de atenção nutricional no pré-natal e puerpério e a integralidade da atenção materno infantil”. Universidade Federal de São Paul; 2014.

Apoio: Conselho Nacional de Desenvolvimento Científico e Tecnológico (Processo nº 486017/2011-7). 
variáveis da dimensão processo: cálculo do índice de massa corporal (35\%), acompanhamento do estado nutricional na curva índice de massa corporal/semana gestacional (46\%) e realização de aconselhamento nutricional individualizado no pré-natal (14\%).

\section{Conclusão}

A atenção nutricional pré-natal e puerperal é insatisfatória para alcançar a integralidade do cuidado. Faz-se necessário sensibilizar gestores locais e capacitar equipes de saúde para assegurar a efetividade de tais ações em Santos.

Palavras-chave: Avaliação em Saúde. Atenção Primária à Saúde. Cuidado pré-natal. Período pós-parto. Programas e políticas de nutrição e alimentação.

\section{A B S T R A C T}

\section{Objective}

To evaluate the perception of managers regarding the nutritional care to prenatal and postpartum in Primary Health Units in Southern, São Paulo, Brazil.

\section{Methods}

It was conducted a census of 28 Primary Health Units from the insular area, using interviews with managers. The nutritional care was assessed according to infrastructure, work process and food and nutrition surveillance.

\section{Results}

The insertion of dietitians was the main insufficient component of dimension structure, interfering negatively on nutritional care for $60 \%$ of managers. It was founded low compliance for the following variables of the process dimension: calculate the body mass index (35\%), monitoring of nutritional status in body mass index/ gestational week curve (46\%) and conducting individualized nutritional counseling in antenatal (14\%).

\section{Conclusion}

Prenatal and postpartum nutritional care is unsatisfactory to achieve comprehensive care. It is necessary to sensitize local manager's awareness and qualify the health teams to ensure the effectiveness of such actions in Santos.

Keywords: Health evaluation. Primary Health Care. Prenatal care. Postpartum period. Nutrition programs and policies.

\section{N T R O D U Ç Ã O}

A atenção ao pré-natal figura como uma das políticas centrais do Sistema Único de Saúde (SUS) no Brasil, contudo, apesar do crescimento da cobertura pré-natal ${ }^{1}$, permanecem dificuldades quanto à qualidade da atenção prestada. Estudos recentes revelam que uma pequena parcela das gestantes inscritas nos programas de pré-natal realiza as ações mínimas preconizadas ${ }^{1-5}$, o que sugere limitações no atendimento prestado pelos serviços de saúde, marcados pela medicalização em detrimento de medidas de prevenção e promoção da saúde 4 .

Para uma atenção ao pré-natal e ao puerpério que promova a saúde materna e neonatal, com acompanhamento precoce, diagnóstico e tratamento adequado de intercorrências, o Ministério da Saúde disponibiliza um conjunto de materiais técnicos e referenciais a ser utilizado pelos serviços ${ }^{6,7}$.

Um dos componentes essenciais para a integralidade da atenção ao pré-natal e ao puerpério é a atenção nutricional, que compreende cuidados de alimentação e nutrição voltados à promoção e à proteção da saúde, prevenção, diagnóstico e tratamento de agravos ${ }^{8}$. Desvios no estado nutricional pré-gestacional e no ganho ponderal materno associam-se ao aumento de riscos para síndrome hipertensiva gestacional, diabetes gestacional ${ }^{9,10}$ e baixo peso ao nascer ${ }^{9,11}$. Estas situações constituem importantes problemas 
de saúde pública que podem ser evitados por adequada atenção pré-natal ${ }^{7}$. Nesse contexto, a vigilância alimentar e nutricional e a atenção nutricional na Atenção Básica permitem identificar fatores de risco e atuar sobre eles ${ }^{12}$, garantindo intervenções na gestação, no período da amamentação e no crescimento/desenvolvimento infantil, além de promover a segurança alimentar e nutricional ${ }^{13}$.

O monitoramento e a avaliação da atenção ao pré-natal são estratégicos para subsidiar decisões nos serviços que facultem o aprimoramento da assistência e a diminuição dos índices de morbimortalidade materna e perinatal ${ }^{3,14}$. Investigações nacionais e internacionais que avaliaram a qualidade desses cuidados, incluindo a atenção nutricional, sinalizaram deficiências no seu processo ${ }^{2,15-20}$. Apesar da evidente relevância, registram-se poucos estudos de avaliação da atenção nutricional ofertada ao pré-natal e ao puerpério ${ }^{15,16}$. Além disso, inexistem dados na literatura referentes ao município de Santos (SP), cujo índice de mortalidade infantil supera a média do estado de São Paulo²1.

Este artigo tem como objetivo avaliar a atenção nutricional à saúde materno infantil durante o pré-natal e o puerpério na rede básica de saúde do SUS do Município de Santos, São Paulo.

\section{M É T O D O S}

Trata-se de estudo censitário das 28 unidades de Atenção Básica em Saúde, realizado na área insular do Município de Santos. A Atenção Básica de Santos possui 28 unidades de saúde - 19 Unidades Básicas de Saúde (UBS) e nove Unidades de Saúde da Família (USF) - distribuídas em 4 regiões que compõem a área insular do município (Orla, Centro, Morros e Zona Noroeste) da seguinte maneira: 6 na região do Centro (5 UBS e uma USF), 6 na Região da Orla (apenas UBS), 8 na Zona Noroeste (5 UBS e 3 USF) e 8 nos Morros (3 UBS e 5 USF). As regiões Centro, Morro e Zona No- roeste apresentam maiores desigualdades socioeconômicas, enquanto a Orla é a região do município que apresenta melhores condições de moradia e infraestrutura ${ }^{22}$. Segundo o Instituto Brasileiro de Geografia e Estatística22, a região insular concentra 99,32\% da população santista, e, por isso, não foram incluídas as unidades da área continental.

Nesses dois tipos de serviços, respeitando-se as particularidades de cada um, as equipes são compostas basicamente por médicos, enfermeiros, técnicos e auxiliares de enfermagem, dentista e agentes comunitários de saúde. Os demais profissionais, incluindo o nutricionista, inserem-se na rede a partir da Seção de Atenção à Saúde da Comunidade, cuja função é oferecer apoio matricial à Atenção Básica.

$O$ atendimento pré-natal de baixo risco é realizado em UBS e USF. De acordo com os últimos dados consolidados para o município, foram registrados 5596 atendimentos de pré-natal na Atenção Básica em 201323; 4908 nascidos vivos no ano de 2012 e uma taxa de mortalidade infantil de 13,9/1 000 nascidos vivos para o mesmo período ${ }^{7}$.

Para avaliação do processo de atenção nutricional no pré-natal e no puerpério, foi utilizado como referencial teórico o modelo de Donabedian ${ }^{24}$ de avaliação dos serviços de saúde. O autor propõe que a avaliação se dê em três níveis: 1) avaliação da estrutura, que demanda, necessariamente, a investigação sobre os recursos físicos, organizacionais e técnicos; 2) avaliação do processo, que implica a análise dos procedimentos entre servidor e consumidor, e 3) avaliação de resultado, que discrimina a situação final de saúde (individual ou coletiva) como resultante da complexa interação entre servidor e consumidor das ações de saúde.

Conforme Donabedian ${ }^{24}$, um bom sistema de avaliação de serviços de saúde deve considerar as dimensões de estrutura, processo e resultado dos serviços prestados. Para o autor, "uma boa estrutura aumenta a probabilidade de um bom 
processo e um bom processo aumenta a probabilidade de um bom desfecho" (p.1147).

Neste estudo, assumiu-se como princípio que a atenção nutricional é componente essencial para alcançar a integralidade da atenção no pré-natal. Verifica-se, entretanto, que ainda que exista uma matriz de ações básicas previstas para esse ciclo da vida, a avaliação da atenção nutricional não foi prevista nos documentos oficiais. Além disso, são escassos os estudos que avaliam a atenção nutricional no pré-natal e no puerpério na Atenção Básica ${ }^{15,18}$. Desse modo, os autores deste artigo construíram um modelo de avaliação de serviços aplicado à atenção nutricional oferta- da ao pré-natal e ao puerpério (Figura 1), que partiu dos problemas gestacionais passíveis de intervenção nutricional no pré-natal e considerou as principais rotinas preconizadas pelo Ministério da Saúde, cuja realização é necessária para a efetividade da atenção nutricional7 .

A construção desse modelo permitiu selecionar as variáveis que compuseram as dimensões de estrutura e processo para a atenção nutricional. Dessa forma, a conformidade dos parâmetros avaliados foi entendida como a consonância da estrutura e do processo ao que é preconizado pelo Ministério da Saúde, uma vez que são padrões mínimos esperados para a assistência de quali-

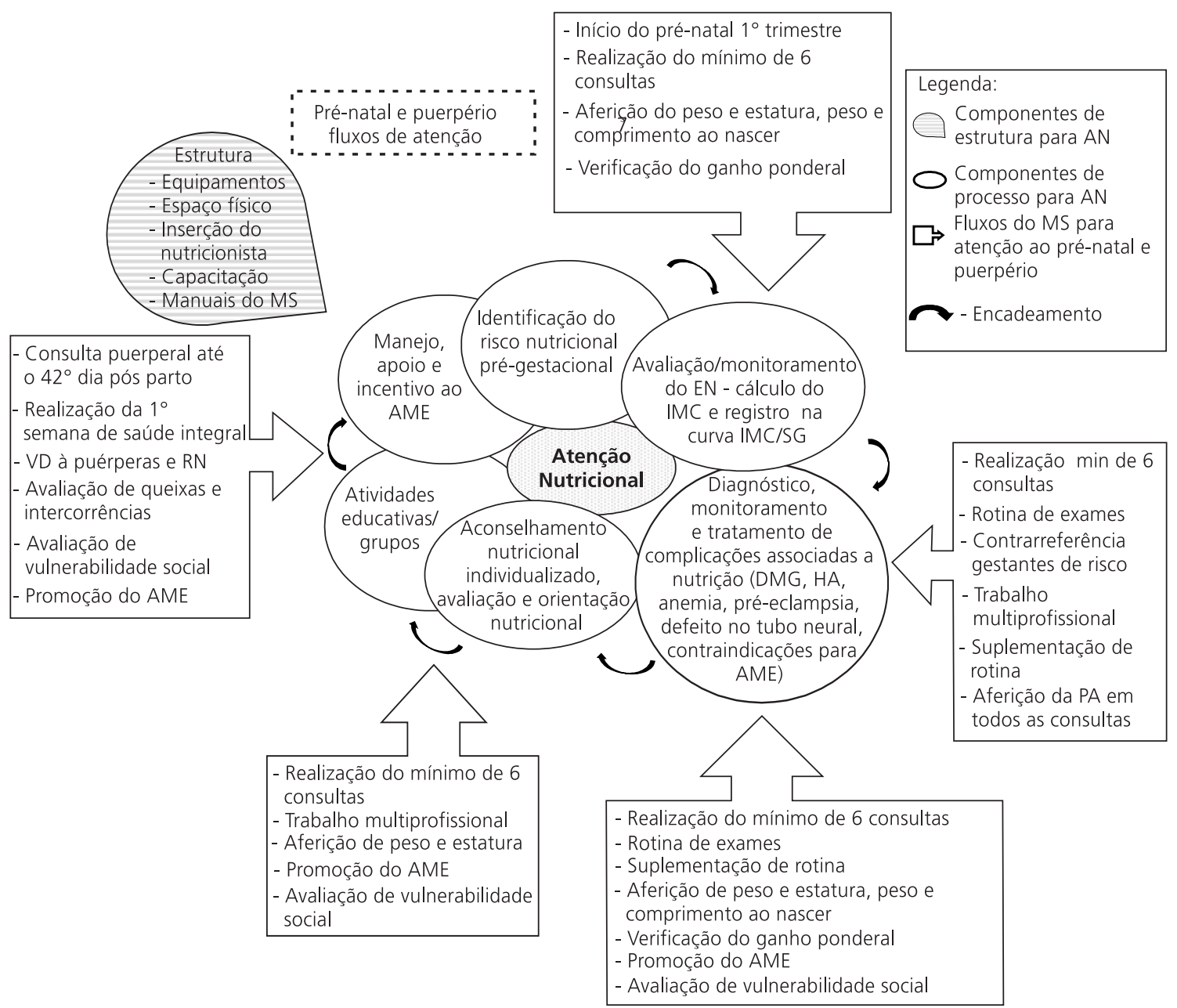

Figura 1. Proposta de modelo de avaliação da atenção nutricional para a integralidade baseado nos componentes das dimensões estrutura e processo. Santos (SP), 2013.

Nota: MS: Ministério da Saúde; EN: Estado Nutricional; AN: Atenção Nutricional; PA: Pressão Arterial; IMC: Índice de Massa Corporal; SG: Semana Gestacional; DMG: Diabetes Mellitus Gestacional; HA: Hipertensão Arterial; AME: Aleitamento Materno Exclusivo; VD: Visita Domiciliar; RN: Recém-Nascido. 
dade e indispensáveis para a integralidade da atenção nutricional25.

As variáveis que compuseram a dimensão estrutura foram: a disponibilidade dos equipamentos de antropometria, sua frequência de manutenção, o espaço físico para as ações de nutrição, a inserção do nutricionista no serviço, a realização de capacitações em antropometria e o manejo dos manuais do Ministério da Saúde.

Para a dimensão processo de trabalho no pré-natal, foram avaliadas as variáveis: tempo de gestação no início do acompanhamento pré-natal, número de consultas realizadas, exames básicos solicitados, suplementação de rotina, realização de trabalho multiprofissional, realização de atividades educativas, avaliação do risco nutricional, realização de avaliação antropométrica, registros em prontuários e consulta nutricional de rotina.

Para a dimensão processo de trabalho no puerpério, as variáveis utilizadas foram: agendamento de consultas e visitas domiciliares para as puérperas e recém-nascidos, avaliação e orientação alimentar da puérpera, suplementação de rotina, avaliação antropométrica dos recém-nascidos, vigilância e realização de ações para a promoção do aleitamento materno exclusivo.

A avaliação consistiu na verificação da conformidade da estrutura das UBS/USF e no processo de atenção nutricional durante o pré-natal, na percepção dos gestores que, nesse caso, correspondem aos gestores locais das unidades de saúde, denominados chefe de seção. A escolha do gestor como fonte das informações deve-se ao seu papel central na tomada de decisões para o alcance das metas estabelecidas, em documentos que norteiam as ações de planejamento em saúde, no âmbito do SUS. Portanto, não foi feita avaliação in loco pela pesquisadora, mas escutou-se a avaliação dos gestores, que puderam consultar documentos internos, como relatórios de produção das várias áreas do serviço e indicadores de vigilância utilizados, como o Sistema de Informação da Atenção Básica e o Sistema de Vigilância Alimentar e Nutricional, bem como outros profissionais ali presentes. As consultas a essas fontes se fizeram necessárias para responder a questões relativas ao início do acompanhamento pré-natal, à realização de exames laboratoriais, ao número de consultas realizadas e ao acompanhamento nutricional.

O instrumento de coleta, elaborado com base em protocolos do Ministério da Saúde ${ }^{6,7,26} \mathrm{e}$ nas diretrizes da Política Nacional de Alimentação e Nutrição (PNAN) ${ }^{8}$, é composto por questões fechadas que contemplam informações sobre os serviços de saúde, como infraestrutura, composição da equipe, processo de trabalho e vigilância alimentar e nutricional, e ainda por questões que permitem analisar a gestão da atenção nutricional, com ênfase no pré-natal e no puerpério. O instrumento foi submetido a pré-teste.

Os gestores foram entrevistados por entrevistadores previamente capacitados a partir do instrumento supracitado. Após os gestores das 28 unidades de saúde assinarem o Termo de Consentimento Livre e Esclarecido, foram entrevistados em suas unidades de trabalho. As entrevistas ocorreram durante o mês de dezembro de 2012, e cada uma teve duração de 60 a 90 minutos.

Os dados foram submetidos a tratamento estatístico, a partir de análises descritivas, contemplando estatísticas frequentistas e medidas de tendência central e de dispersão para caracterizar os serviços de saúde e as ações de alimentação e nutrição realizadas. Para a análise estatística dos dados foram utilizados os softwares Epi Info (Center for Disease Control and Prevention, Atlanta, Geórgia, Estados Unidos) versão 3.5.3 e o Statistical Package for the Social Sciences (SPSS Inc., Chicago, Illinóis, Estados Unidos) versão 16. O estudo foi aprovado pelo Comitê de Ética em Pesquisa da Universidade Federal de São Paulo, sob o Parecer n³ 32.900.

\section{RES U LTA DOS}

Foram entrevistados 28 gestores das unidades de saúde de Santos: 24 do sexo feminino e 4 do sexo masculino. A média de idade dos entre- 
vistados foi 41,6 anos, Desvio-Padrão-DP=8,8, e o tempo médio de trabalho na unidade foi de $45,3$ meses ( $D P=38,3)$. Quanto à formação, a maioria dos profissionais entrevistados $(n=20)$ era enfermeiro, os demais tinham formação em odontologia $(n=3)$, serviço social $(n=2)$, psicologia $(n=2)$ e farmácia $(n=1)$.

Na Tabela 1 estão representados os dados da dimensão estrutura em conformidade para a realização da atenção nutricional. Com relação aos equipamentos para avaliação antropométrica, encontrou-se baixa conformidade apenas para a disponibilidade de balança portátil nas UBS. Para os demais equipamentos, mais de $89,0 \%$ das unidades avaliadas apresentaram conformidade. A avaliação dos gestores com relação à frequência de manutenção dos equipamentos foi ruim em sua maioria (42,8\%), seguida por regular $(35,7 \%)$ e boa $(17,8 \%)$.

Com relação ao espaço físico, a conformidade para ações educativas e atividades em grupos foi a variável com pior resultado. Apenas $26,3 \%$ das UBS e $33,3 \%$ das USF apresentaram adequação. Nas demais variáveis de espaço físico, as UBS apresentaram maior proporção de conformidade quando comparadas às USF. Apesar da alta proporção de conformidade dos equipamentos para avaliação antropométrica, principalmente nas USF (100,0\%), a adequação do espaço físico para essa prática apresentou conformidade em $73,7 \%$ das UBS e em $55,6 \%$ das USF. Com relação ao manejo dos manuais técnicos do Ministério da Saúde, 78,9\% das UBS e 88,9\% da USF apresentaram conformidade com relação à existência e utilização do "Manual técnico de pré-natal e puerpério" e 62,3 e 77,8\% apresentaram conformidade com relação ao "Caderno de Atenção Básica: saúde da criança: nutrição infantil, aleitamento materno e alimentação complementar", respectivamente, para UBS e USF.

Identificou-se a percepção dos gestores quanto à inserção do nutricionista e à oferta de capacitação em antropometria, ambas as variáveis da dimensão estrutura. Das 28 unidades de saúde avaliadas, a inserção do nutricionista se dá em apenas 13 UBS $(68,4 \%)$ e em 6 USF $(66,6 \%)$, das quais 12 UBS contam com apenas um período de atuação desse profissional e uma UBS com dois períodos. Das 6 USF, 4 contam com um período e duas com dois períodos. A maior parte dos gestores avaliou como insuficiente a inserção de nutricionistas na rede básica de Santos (Tabela 2).

Tabela 1. Avaliação de conformidade da estrutura de unidades de saúde para atenção ao pré-natal e ao puerpério por tipo de serviço, segundo percepção dos gestores. Santos (SP), 2013 ( $n=28)$.

\begin{tabular}{|c|c|c|c|c|}
\hline \multirow{3}{*}{ Estrutura } & \multicolumn{4}{|c|}{ Conformidade } \\
\hline & \multicolumn{2}{|c|}{ UBS $(n=19)$} & \multicolumn{2}{|c|}{ USF $(n=9)$} \\
\hline & $\mathrm{n}$ & $\%$ & $\mathrm{n}$ & $\%$ \\
\hline Disponibilidade de balança plataforma & 18 & 94,7 & 9 & 100,0 \\
\hline Disponibilidade de balança pediátrica & 19 & 100,0 & 9 & 100,0 \\
\hline Disponibilidade de antropômetro vertical & 17 & 89,5 & 9 & 100,0 \\
\hline Disponibilidade de antropômetro horizontal & 19 & 100,0 & 9 & 100,0 \\
\hline Disponibilidade de fita antropométrica & 19 & 100,0 & 9 & 100,0 \\
\hline Disponibilidade de balança portátil & 3 & 15,8 & 9 & 100,0 \\
\hline Espaço físico adequado para ações educativas/grupos & 5 & 26,3 & 3 & 33,3 \\
\hline Espaço físico adequado para interconsultas e discussões de caso & 13 & 68,4 & 3 & 33,3 \\
\hline Espaço físico adequado para antropometria & 14 & 73,7 & 5 & 55,6 \\
\hline Espaço físico adequado para reuniões de equipe & 12 & 63,2 & 4 & 44,4 \\
\hline Manejo do "Manual Pré-natal e puerpério: atenção qualificada e humanizada" & 15 & 78,9 & 8 & 88,9 \\
\hline $\begin{array}{l}\text { Manejo do "Caderno de Atenção Básica: saúde da criança: nutrição infantil, } \\
\text { aleitamento materno e alimentação complementar" }\end{array}$ & 12 & 63,2 & 7 & 77,8 \\
\hline
\end{tabular}

Nota: UBS: Unidade Básica de Saúde; USF: Unidade de Saúde da Família. 
Tabela 2. Avaliação de conformidade do processo de atenção ao pré-natal, por tipo de serviço, segundo percepção dos gestores. Santos (SP), $2013(n=28)$.

\begin{tabular}{|c|c|c|c|c|}
\hline \multirow{3}{*}{ Processo pré-natal } & \multicolumn{4}{|c|}{ Conformidade } \\
\hline & \multicolumn{2}{|c|}{ UBS $(n=19)$} & \multicolumn{2}{|c|}{$\operatorname{USF}(n=9)$} \\
\hline & $\mathrm{n}$ & $\%$ & $\mathrm{n}$ & $\%$ \\
\hline Início no primeiro trimestre & 19 & 100,0 & 9 & 100,0 \\
\hline Realização do mínimo de 6 consultas & 19 & 100,0 & 9 & 100,0 \\
\hline Trabalho em equipe multiprofissional & 17 & 89,5 & 9 & 100,0 \\
\hline Realização de atividades educativas & 15 & 78,9 & 8 & 88,9 \\
\hline Identificação do risco nutricional & 18 & 94,7 & 8 & 88,9 \\
\hline Contrarreferência das gestantes encaminhadas & 13 & 68,4 & 8 & 88,9 \\
\hline \multicolumn{5}{|l|}{ Avaliação/monitoramento do estado nutricional } \\
\hline Aferição do peso em todas as consultas & 19 & 100,0 & 9 & 100,0 \\
\hline Aferição da pressão arterial em todas as consultas & 19 & 100,0 & 9 & 100,0 \\
\hline Aferição da estatura & 19 & 100,0 & 8 & 88,9 \\
\hline Cálculo do IMC & 5 & 26,3 & 5 & 55,6 \\
\hline Acompanhamento no cartão da gestante & 18 & 94,7 & 9 & 100,0 \\
\hline Registro no prontuário & 19 & 100,0 & 9 & 100,0 \\
\hline Preenchimento da curva IMC/semana gestacional & 8 & 42,1 & 5 & 55,6 \\
\hline Aconselhamento nutricional individual na rotina do pré-natal & 4 & 21,1 & 0 & 0,0 \\
\hline \multicolumn{5}{|l|}{ Exames laboratoriais } \\
\hline Glicemia de jejum (2x durante a gestação) & 15 & 78,9 & 8 & 88,9 \\
\hline Urina tipo 1 ( 2 x durante a gestação) & 15 & 78,9 & 7 & 77,8 \\
\hline Hemograma & 17 & 89,5 & 9 & 100,0 \\
\hline Testagem anti-HIV & 19 & 100,0 & 9 & 100,0 \\
\hline Sorologia para hepatite & 19 & 100,0 & 9 & 100,0 \\
\hline \multicolumn{5}{|l|}{ Suplementação de rotina } \\
\hline Sulfato ferroso & 18 & 94,7 & 8 & 88,9 \\
\hline Ácido fólico & 18 & 94,7 & 8 & 88,9 \\
\hline
\end{tabular}

Nota: UBS: Unidade Básica de Saúde; USF: Unidade de Saúde da Família; IMC: Índice de Massa Corporal.

Em relação à conformidade para os critérios de processo durante o pré-natal, segundo os gestores, todas as unidades avaliadas apresentaram conformidade quanto às variáveis início de pré-natal no primeiro trimestre e realização do mínimo de seis consultas. As demais variáveis apresentaram maior proporção de conformidade para as USF em relação às UBS, com exceção dos procedimentos identificação do risco nutricional, aferição da estatura, consulta nutricional como rotina do pré-natal e suplementação de rotina, que apresentaram proporção discretamente maior de conformidade para as UBS (Tabela 2).

Além da baixa proporção de unidades de saúde que realizam consulta nutricional na rotina do pré-natal, chama atenção a baixa proporção de adequação das UBS e USF quanto à realização dos procedimentos necessários ao acompanhamento do estado nutricional, como o cálculo do Índice de Massa Corporal (IMC) e o preenchimento da curva IMC/semana gestacional, segundo recomendação oficial? .

A maioria dos procedimentos preconizados pelo Ministério da Saúde ${ }^{7}$ para o período puerperal foi realizada em maior proporção pelas USF (Tabela 3). Com relação às ações de atenção à saúde da puérpera e do recém-nascido, menos de $50 \%$ das UBS realizaram: visitas domiciliares na primeira semana após o parto tanto para as puérperas em geral quanto para os recém-nascidos de risco, agendamento entre o $7^{\circ}$ e $10^{\circ}$ dia após o parto para a Primeira Semana de Saúde Integral, avaliação da vulnerabilidade social e da alimentação da puérpera. Para as USF, a variável que apresentou conformidade em menos de $50 \%$ 
Tabela 3. Avaliação de conformidade do processo da atenção ao puerpério, por tipo de serviço, segundo percepção dos gestores. Santos (SP), $2013(n=28)$.

\begin{tabular}{|c|c|c|c|c|}
\hline \multirow{3}{*}{ Processo puerpério } & \multicolumn{4}{|c|}{ Conformidade } \\
\hline & \multicolumn{2}{|c|}{ UBS $(n=19)$} & \multicolumn{2}{|c|}{ USF $(n=9)$} \\
\hline & $\mathrm{n}$ & $\%$ & $\mathrm{n}$ & $\%$ \\
\hline \multicolumn{5}{|l|}{ Atenção à saúde da puérpera e recém-nascido } \\
\hline Busca ativa agente comunitário de saúde para agendamento de consultas & 15 & 78,9 & 7 & 77,8 \\
\hline Visita domiciliar nas primeiras semanas após o parto a todas as puérperas & 9 & 47,4 & 6 & 66,7 \\
\hline Visita domiciliar nas primeiras semanas após o parto apenas aos recém-nascidos de risco & 8 & 42,1 & 4 & 44,4 \\
\hline Agendamento de consulta de puerpério até $42^{\circ}$ dia pós-parto & 17 & 89,5 & 9 & 100,0 \\
\hline Agendamento da primeira consulta do recém-nascido & 19 & 100,0 & 9 & 100,0 \\
\hline Realização entre o $7^{\circ}$ e $10^{\circ}$ dia após o parto da Primeira Semana de Saúde Integral & 3 & 15,8 & 5 & 55,6 \\
\hline Avaliação de queixas e intercorrências & 18 & 94,7 & 9 & 100,0 \\
\hline Avaliação de vulnerabilidade social & 8 & 42,1 & 6 & 66,7 \\
\hline Avaliação alimentar e nutricional da puérpera & 8 & 42,1 & 7 & 77,8 \\
\hline Orientação sobre alimentação da puérpera & 17 & 89,5 & 9 & 100,0 \\
\hline Prescrição de ferro até os três meses pós-parto & 12 & 63,2 & 8 & 88,9 \\
\hline \multicolumn{5}{|l|}{ Primeira consulta do recém-nascido } \\
\hline Verificação de peso ao nascer e peso atual & 19 & 100,0 & 8 & 88,9 \\
\hline Verificação do comprimento & 19 & 100,0 & 8 & 88,9 \\
\hline Cálculo do ganho ponderal diário & 17 & 89,5 & 6 & 66,7 \\
\hline Identificação do padrão de aleitamento & 19 & 100,0 & 8 & 88,9 \\
\hline \multicolumn{5}{|l|}{ Promoção do Aleitamento Materno Exclusivo } \\
\hline Ações educativas para incentivo ao Aleitamento Materno Exclusivo em grupo & 16 & 84,2 & 9 & 100,0 \\
\hline Ações para incentivo ao Aleitamento Materno Exclusivo em consulta individual & 18 & 94,7 & 9 & 100,0 \\
\hline Ações para incentivo ao Aleitamento Materno Exclusivo em visitas domiciliares & 13 & 68,4 & 8 & 88,9 \\
\hline
\end{tabular}

Nota: UBS: Unidade Básica de Saúde; USF: Unidade de Saúde da Família.

foi a visitas domiciliares na primeira semana para os recém-nascidos de risco.

\section{I S C U S S Ã O}

Este estudo teve por objetivo avaliar a atenção nutricional ofertada ao pré-natal e ao puerpério pela rede básica do SUS do Município de Santos, segundo os componentes estrutura e processo, a partir de um censo das unidades de saúde. Para Donabedian ${ }^{24}$, a dimensão estrutura é representada por insumos, força de trabalho e estrutura organizacional, que, quando adequados, garantem um bom processo, definido por cuidados oferecidos pelos profissionais e recebidos pelos usuários a fim de favorecer um bom desfecho ${ }^{24}$. Como diferencial para essa avaliação, partiu-se da premissa de que a atenção nutricional é requisito para a integralidade do cuidado.
Estudos recentes se dedicaram a avaliar a qualidade do pré-natal ${ }^{2,15,18,26}$ por meio do modelo proposto por Donabedian ${ }^{24}$, contudo não foram identificadas investigações que avaliaram a atenção nutricional no pré-natal e no puerpério na Atenção Básica, considerando suas dimensões de estrutura e processo. Destaca-se, neste estudo, a construção de um modelo de avaliação de serviços aplicado à atenção nutricional ao pré-natal e ao puerpério, tendo com base as ações de saúde previstas pelo Ministério da Saúde, o que ressalta o seu caráter inovador.

No geral, identificaram-se proporções de conformidade superiores às observadas na literatura, possivelmente pela metodologia utilizada. As respostas das entrevistas com os gestores podem ter sofrido vieses de informações e ter sido supervalorizadas pelo conhecimento prévio dos gestores sobre as recomendações para o pré-natal e puerpério, o que configura uma limitação do 
estudo. Estes limites poderiam ser minimizados por estratégias de observação direta, observação participante ou por estudo longitudinal. Em pesquisa com metodologia semelhante, revelou-se que gestores e profissionais tendem a responder índices mais elevados, sendo os gestores os que emitem melhores opiniões sobre as dimensões desses serviços ${ }^{27}$.

Com relação à estrutura das unidades, não existe padronização para esse tipo de avaliação: usualmente são utilizados documentos oficiais e portarias do Ministério da Saúde, como o "Caderno de Atenção Básica: pré-natal de baixo risco"7, o "Manual de estrutura física das unidades básicas de saúde" 28 e a "Política nacional de atenção básica"29. Prevê-se que as unidades de saúde disponham de equipamentos e materiais adequados ao elenco de ações, além de salas para reuniões e educação em saúde 28,29

Durante o pré-natal e o puerpério, gestantes e crianças precisam ter seu peso e estatura/ comprimento aferidos com frequência para garantir o bom acompanhamento do estado nutricional ${ }^{7}$. Com relação aos equipamentos mínimos para isso, duas UBS não dispunham de antropômetro vertical, o que inviabiliza a aferição da estatura de adultos e o acompanhamento do estado nutricional. Além disso, a manutenção dos equipamentos, avaliada como ruim pela maioria dos gestores, é condição para viabilizar a avaliação antropométrica. Sobre o espaço físico para antropometria, não há recomendação específica, e os equipamentos podem estar dispostos nos consultórios médicos. Apesar disso, nove gestores não consideraram ter espaço adequado para essa prática.

A adequação do espaço físico para reuniões, ações educativas e discussões de caso foi ainda mais insatisfatória na percepção dos gestores. O Ministério da Saúde preconiza ações educativas em grupo ou individualmente durante todo o período de acompanhamento. Os resultados chamam a atenção para a baixa percentagem de adequação do espaço físico para essas atividades: menos de $30 \%$ dos gestores consideraram adequados, embora cerca de $80 \%$ tenham afirmado realizar essas atividades em suas unidades.

O presente estudo também verificou a percepção dos gestores quanto à oferta de capacitação em antropometria: 85,7\% avaliaram tal oferta como insuficiente. Esse resultado alerta para a necessidade de ações de alimentação e nutrição na Atenção Básica que prescindem da atuação do nutricionista propriamente dita, corroborando achados de Camossa et al. ${ }^{30}$ que demonstraram ser suficiente a presença do nutricionista para capacitar os profissionais da equipe, sugerindo o formato de apoio matricial.

Na avaliação da dimensão estrutura, também foi encontrada baixa inserção do nutricionista na Atenção Básica do município. Santos conta com apenas 3 nutricionistas que atuam nas áreas insular e continental. Na área insular, trabalham entre um e dois períodos de quatro horas semanais em cada uma das 19 unidades em que estão inseridas. Das 28 unidades de Saúde avaliadas, 6 UBS e 3 USF não têm nutricionista. Apesar dessa realidade, em mais da metade $(n=11)$ das unidades que contam com atuação do nutricionista, os gestores avaliaram ser suficiente sua inserção, possivelmente por desconhecimento da sua potencial contribuição na qualificação das ações da equipe de saúde na Atenção Básica.

As ações de alimentação e nutrição recomendadas para o pré-natal e puerpério estão descritas e disponibilizadas para os gestores da Atenção Básica ${ }^{7,26}$. Contudo, aproximadamente 20 e $30 \%$ dos gestores das UBS e USF, respectivamente, afirmaram não conhecer ou utilizar esses materiais, fato que pode demonstrar o não reconhecimento do papel dessas ações destinadas ao pré-natal e ao puerpério.

A avaliação da adequação do pré-natal tem sido objetivo de estudos recentes. Considerando-se os parâmetros mínimos preconizados pelo Programa de Humanização no Pré-natal e Nascimento (PHPN) - início precoce de acompanhamento e realização do mínimo de seis consultas -, os resultados da dimensão processo deste estudo superaram os achados por outros tra- 
balhos. Domingues et al. ${ }^{5}$ constataram o início tardio do pré-natal em $25 \%$ das gestantes avaliadas no município do Rio de Janeiro, o que interferiu no número de consultas daquelas que iniciaram com mais de 16 semanas. Em outro estudo, realizado por Vettore et al. ${ }^{31}$, verificou-se inadequação em 14\% das gestantes avaliadas com o índice de Kotelchuck, classificação adotada quando realizadas menos de $50 \%$ das consultas esperadas e início após o quarto mês de gestação. Anversa et al. ${ }^{32}$ também identificaram um baixo percentual de adequação (59\%) do pré-natal no município de Santa Maria (RS), quando avaliados o início do pré-natal antes de 20 semanas e o número mínimo de seis consultas. Baixa adequação da cobertura pré-natal também foi identificada na literatura internacional20,33. Destaca-se que, nos estudos citados, realizou-se pesquisa documental a partir dos registros em prontuário e cartão da gestante, enquanto no presente estudo, que apontou conformidade para esses dois critérios em $100 \%$ dos casos, avaliou-se a percepção dos gestores sobre a adequação. Essa escolha pode sugerir uma superestimação dos valores encontrados, uma vez que tais critérios de adequação são amplamente reconhecidos pelos gestores e constantemente objeto de avaliação.

No que tange aos cuidados nutricionais nessa fase da vida, este estudo apresentou resultados bastante insatisfatórios para os critérios cálculo do IMC, preenchimento da curva IMC/ semana gestacional e realização de aconselhamento nutricional como rotina do pré-natal. Apesar desses resultados, cerca de $90 \%$ dos gestores entrevistados afirmaram realizar a identificação do risco nutricional e a aferição da pressão arterial, peso e estatura em todas as consultas. A conformidade dos critérios preconizados pelo PHPN tem-se mostrado crescente, de acordo com a última Pesquisa Nacional de Demografia e Saúde da Criança e da Mulher ${ }^{1}$, no entanto, com relação ao conjunto das ações mínimas recomendadas pelo programa para a atenção de qualidade, a literatura identificou deficiências no cuidado ofertado ao pré-natal e puerpério.
Chama a atenção, neste estudo, o paradoxo entre a alta proporção de identificação do risco nutricional e a baixa proporção do cálculo de IMC, relatadas pelos gestores. O Ministério da Saúde ${ }^{7}$ preconiza a avaliação e o acompanhamento do estado nutricional da gestante para diagnosticar o risco nutricional, verificado pelo cálculo do IMC (peso/altura²). Dessa forma, sugere-se que os gestores, ao afirmarem alta adequação da identificação do risco nutricional, referem-se à aferição isolada do ganho de peso gestacional, cuja avaliação de adequação foi de 100\%, segundo eles.

Niquini et al. ${ }^{15}$, ao avaliarem o processo da atenção nutricional no pré-natal em 7 USF do Rio de Janeiro, identificaram resultados semelhantes. Dentre as 230 gestantes avaliadas, cerca de $90 \%$ apresentavam conformidade do número de registro de peso por número de consulta no cartão de pré-natal; em contrapartida, 90\% dos cartões não tinham qualquer marcação no gráfico de IMC/semana gestacional. Estes dados reforçam os achados do presente estudo sobre a importância dada pelos profissionais ao ganho de peso da gestante como critério isolado para identificar riscos nutricionais, independentemente da avaliação do estado nutricional, que engloba cálculo do IMC e preenchimento do gráfico de IMC/semana gestacional.

A deficiência na realização do aconselhamento nutricional como rotina do pré-natal, demonstrada neste estudo, reflete no acompanhamento deficiente do estado nutricional durante a gestação, impossibilitando o monitoramento e as intervenções necessárias. Segundo a literatura, a inadequação no estado nutricional pré-gestacional apresenta proporções elevadas no Brasil, sendo frequente em mais de $40 \%$ das gestantes brasileiras usuárias do SUS ${ }^{31,34,35}$, bem como em outros países ${ }^{19,36,37}$, o que constitui problema de saúde pública e merece atenção pré-natal qualificada, incluindo a vigilância alimentar e nutricional $^{7}$.

Este estudo encontrou deficiências no cuidado - uma vez que não são realizados o 
monitoramento do estado nutricional nem as ações promotoras de saúde passíveis de atuar sobre os desvios nutricionais e fomentar melhores desfechos à mãe e ao bebê -, o que representa uma lacuna para o alcance da integralidade da atenção ao pré-natal.

A alta proporção de conformidade da realização dos exames laboratoriais no início e no final da gestação supera os achados de outros estudos que demonstraram proporções de conformidade abaixo de 50\% ${ }^{2,5,32}$. Resultados superiores aos da literatura também foram encontrados para a suplementação de rotina de ferro e ácido fólico ${ }^{15}$.

Quanto aos procedimentos do puerpério, os resultados desta investigação exibem uma proporção maior de conformidade para as ações destinadas ao cuidado com os recém-nascidos, o que parece reforçar a desvinculação entre a saúde da mãe e a do recém-nascido, e a transferência do cuidado da saúde materna para a saúde da criança em detrimento do olhar para a saúde da mulher $^{38}$. A necessidade da consulta puerperal é reconhecida pelos gestores de saúde, o que explica a alta proporção de adequação encontrada neste estudo. Entretanto, tal reconhecimento não tem garantido boa cobertura desse procedimento, conforme demonstrou Parada ${ }^{2}$, que encontrou registros de realização da consulta puerperal em $58,7 \%$ dos prontuários das gestantes avaliadas.

Os achados sobre avaliação alimentar e nutricional da puérpera apresentaram-se insatisfatórios, assim como os encontrados no pré-natal. Destaca-se também a baixa proporção de conformidade da avaliação da vulnerabilidade social, o que aponta fragilidades na integralidade do cuidado. A avaliação alimentar e nutricional também não foi relatada por estudos que avaliaram a atenção pré-natal e puerperal2,39.

No que tange aos procedimentos destinados aos recém-nascidos no puerpério, observou-se maior proporção de conformidade, inclusive, para a avaliação do estado nutricional. A discrepância entre a proporção de conformidade de aferição do peso e do cálculo do ganho ponderal diário dos recém-nascidos parece evidenciar o subestimado papel desse indicador no acompanhamento do estado nutricional nos primeiros dias de vida da criança.

No presente estudo, encontrou-se alta proporção de conformidade para as ações de promoção do Aleitamento Materno Exclusivo, com resultados menos favoráveis para as visitas domiciliares nas UBS. Revisões sistemáticas que subsidiaram a Política Pública de Promoção à Amamentação demonstraram que dentre as estratégias com evidência empírica de efetividade na promoção do aleitamento materno estão os grupos de pré-natal, as visitas domiciliares, os grupos de mães e as sessões individuais conduzidas no pré e pós-natal, os quais proporcionam o diálogo e a escuta, elementos centrais da educação alimentar e nutricional ${ }^{40,41}$.

Com relação à atenção nutricional no período pós-parto, os poucos trabalhos relativos à saúde da puérpera apontaram a importância de ações voltadas à promoção e ao incentivo ao aleitamento materno, o que reforça o hiato existente entre o cuidado à saúde da gestante e o cuidado com o recém-nascido após o parto.

Proporções ainda mais baixas de conformidade foram encontradas para a realização de visitas domiciliares às puérperas nas primeiras semanas pós-parto. No contexto da integralidade do cuidado, as visitas domiciliares são consideradas instrumento importante de proteção à saúde e precisam ser frequentes a fim de estreitar o vínculo entre gestante/nutriz e unidade de saúde a partir da abordagem integral da mulher e sua família em seu contexto social ${ }^{7}$. Para o apoio à amamentação, é ideal que a visita à nutriz ocorra até o $14^{\circ}$ dia após o parto, visto ser este um período de dificuldades e novidades para a mãe, o que pode resultar no desmame precoce ${ }^{42}$.

Embora não tenha sido encontrada grande diferença dos parâmetros avaliados entre UBS e USF, talvez pelo tamanho reduzido do universo das unidades avaliadas, a maior proporção de conformidade foi encontrada nas USF, corroborando 
achados de outros estudos que compararam a atenção ao pré-natal em diferentes tipos de serviço de Atenção Básica ${ }^{32,39}$. A necessidade de acompanhamento puerperal precoce é reforçada pelo estudo de Parada ${ }^{43}$, que relata associação significante entre a ocorrência de dificuldades no início do aleitamento e menores prevalências de Aleitamento Materno Exclusivo.

De maneira geral, o componente de estrutura mais apontado pelos gestores como insuficiente foi a inserção do nutricionista na atenção básica do município, o que pode interferir negativamente na realização dos demais componentes de processo e, portanto, nas ações de alimentação e nutrição ofertadas, tanto pela limitada atuação desse profissional propriamente dita, quanto pela provável insuficiência do apoio matricial dos gestores.

Camossa et al. ${ }^{30}$ avaliaram as representações sociais de diversos profissionais das equipes de saúde da família de um município do estado de São Paulo. Observaram-se visões bastante restritas do trabalho do nutricionista, como o "profissional que passa dieta" ou que "faz o paciente perder peso". Outro aspecto relevante verificado por Camosa et al. ${ }^{30}$ e por Pádua \& Boog ${ }^{44}$ foi a realização de orientações alimentares por outros profissionais, em geral o médico, que as fazem, na maioria das vezes, de forma restritiva e impositiva. Em ambos os estudos, foi possível observar a falta de preparo e domínio de profissionais para abordar os problemas alimentares. Camossa et al. ${ }^{30}$ salienta que muitos profissionais não reconhecem as especificidades do nutricionista e percebem o seu trabalho, muitas vezes, com a finalidade de poupar tempo e dar suporte ao trabalho do médico. O Conselho Federal de Nutricionistas reconhece como atribuições específicas do nutricionista a atenção nutricional especializada e a educação nutricional. Sendo assim, os serviços públicos de saúde não poderiam prescindir desse profissional45.

Este estudo apresenta elementos inovadores tanto por se tratar de censo das unidades de saúde de Santos, retratando a realidade da atenção nutricional da atenção básica no município, quanto por ter sido feita avaliação sob a ótica dos gestores locais, o que possibilita inferir o reconhecimento desses sujeitos no processo de gestão da atenção nutricional na Atenção Básica.

Foi possível visualizar o desconhecimento dos gestores quanto às diretrizes da PNAN, assim como sobre a complexidade da atenção nutricional nas políticas de atenção à saúde materno infantil. A baixa conformidade do acompanhamento do estado nutricional gestacional, a insuficiência das atividades de educação em saúde e a ausência de aconselhamento nutricional na rotina do pré-natal verificadas nesta pesquisa precisam ser tomadas como um alerta para os gestores da Atenção Básica de Santos.

A atenção nutricional nessa fase do curso da vida é componente essencial para o alcance da atenção integral, visto que muitos fatores de risco para a morbimortalidade materno infantil, como baixo peso ao nascer, síndrome hipertensiva gestacional e diabetes gestacional, estão diretamente relacionados ao estado nutricional da gestante e, portanto, necessitam de intervenção nutricional no pré-natal ${ }^{9-11}$.

Por fim, conclui-se que, para a integralidade do cuidado à saúde materno infantil, faz-se necessário sensibilizar gestores da atenção básica e da Secretaria de Saúde do município quanto aos demais níveis de atenção a fim de garantir a efetividade das ações de alimentação e nutrição no pré-natal e no puerpério. Os achados desta investigação podem contribuir para repensar as políticas públicas de Santos com vistas a contribuir para a redução da mortalidade infantil no município.

\section{COLABORADORES}

ASCL PINFILDI contribuiu com a concepção do estudo, a análise e a discussão dos dados e com a redação do manuscrito. LTO ZANGIROLANI, N SPINA e PA MARTINS contribuíram com a concepção do estudo e a discussão dos resultados. MAT MEDEIROS contribuiu com a concepção do estudo, a análise, a discussão dos resultados e com a revisão final do manuscrito. 


\section{REFERÊ NCIAS}

1. Brasil. Ministério da Saúde. Pesquisa Nacional de Demografia e Saúde da Criança e da Mulher: PNDS 2006: dimensões do processo reprodutivo e da saúde da criança. Brasilia: Ministério da Saúde; 2009

2. Parada CMGDL. Avaliação da assistência pré-natal e puerperal desenvolvidas em região do interior do Estado de São Paulo em 2005. Rev Bras Saúde Matern Infant. 2008; 8(1):113-24. http://dx.doi. org/10.1590/S1519-38292008000100013

3. Gonçalves CV, Cesar JA, Mendoza-Sassi RA. Quality and equity in prenatal care: A population- based study in Southern Brazil. Cad Saúde Pública. 2009; 25(11):2507-16. http://dx.doi.org/10.1590/S0102-3 $11 \times 2009001100020$

4. Victora CG, Aquino EM, Do Carmo Leal M, Monteiro CA, Barros FC, Szwarcwald CL. Maternal and child health in Brazil: Progress and challenges. Lancet. 2011; 377(9780):1863-76. http://dx.doi. org/10.1016/S0140-6736(11)60138-4

5. Domingues RMSM, Hartz ZMA, Dias MAB, Leal MC. Avaliação da adequação da assistência pré-natal na rede SUS do Município do Rio de Janeiro, Brasil. Cad Saúde Pública. 2012; 28(3):425-37. http://dx. doi.org/10.1590/S0102-311X2012000300003

6. Brasil. Portaria $n^{\circ}$ 569, de 1 de Junho de 2000. Institui o Programa de Humanização no Pré-natal e Nascimento no âmbito do SUS. Diário Oficial da União. 2000; 8 jun.

7. Brasil. Ministério da Saúde. Secretaria de Atenção à Saúde. Atenção ao pré-natal de baixo risco. Cadernos de Atenção Básica, n 32. Brasília: Ministério da Saúde; 2012.

8. Brasil. Ministério da Saúde. Secretaria de Atenção à Saúde. Política nacional de alimentação e nutrição. Brasília: Ministério da Saúde; 2012.

9. Padilha PDC, Saunders C, Machado RCM, Silva CL, Bull A, Sally EDOF, et al. Associação entre o estado nutricional pré-gestacional e a predição do risco de intercorrências gestacionais. Rev Bras Ginecol Obstet. 2007; 29(10):511-8. http://dx.doi.org/ 10.15 90/S0100-72032007001000004

10. Bertoldi L, Inês M, Bartholow B, Costa S, Teresinha E, Margarida M, et al. Nutritional status of pregnant women: Prevalence and associated pregnancy outcomes. Rev Saúde Pública. 2001; 35(6):502-7. http://dx.doi.org/10.1590/S0034-89102001000 600002

11. Santos MMADS, Baião MR, Barros DC, Pinto ADA, Pedrosa PLM, Saunders C. Estado nutricional pré-gestacional, ganho de peso materno, condições da assistência pré-natal e desfechos perinatais adversos entre puérperas adolescentes. Rev Bras
Epidemiol. 2012; 15(1):143-54. http://dx.doi.org/ 10.1590/S1415-790X20/2000100013

12. Medeiros MAT. Desafios do campo da alimentação e nutrição na Atenção Básica. In: Garcia RWD \& Cervato-Mancuso AM, editores. Mudanças alimentares e educação nutricional. Rio de Janeiro: Guanabara Koogan; 2011. p.173-80.

13. Brasil. Ministério da Saúde. Portaria n 154, de 24 de janeiro de 2008. Cria os Núcleos de Apoio à Saúde da Família NASF. Diário Oficial da União; 2008; 4 mar, p.38-42, Seção 1.

14. Silva AMR, Almeida MF, Matsuo T, Soares DA. Fatores de risco para nascimentos pré-termo em Londrina, Paraná, Brasil. Cad Saúde Pública. 2009; 25(10):2125-38. http://dx.doi.org/10.1590/S0102-3 $11 \times 2009001000004$

15. Niquini RP, Bittencourt SA, Lacerda EMDA, Saunders C, Leal MDC. Avaliação do processo da assistência nutricional no pré-natal em sete unidades de saúde da família do Município do Rio de Janeiro. Ciênc Saúde Colet. 2012; 17(10):2805-16. http://dx.doi.org/10.1590/S1413-812320120010 00028

16. Niquini RP, Bittencourt SA, Lacerda EMDA, Saunders C, Leal MDC. Avaliação da estrutura de sete unidades de saúde da família para a oferta da assistência nutricional no pré-natal no município do Rio de Janeiro, Brasil. Rev Bras Saúde Mater Infant. 2010; 10:61-8. http://dx.doi.org/10.1590/S 1519-3829 2010000500006

17. Santos LA Dos, Mamede FV, Clapis MJ, Bernardi JVB. Nutritional guidance during prenatal care in public health services in Ribeirão Preto: Discourse and care practice. Rev Latino-Am Enfermagem. 2006; 14(5):688-94. http://dx.doi.org/10.1590/ S0104-11692006000500008

18. Costa GD, Cotta RMM, Reis JR, Siqueira-Batista R, Gomes AP, Franceschini SDCC. Avaliação do cuidado à saúde da gestante no contexto do Programa Saúde da Família. Ciênc Saúde Colet. 2009; 14(Supl. 1):1347-57. http://dx.doi.org/10.1590/S14 13-81232009000800007

19. Garmendia ML, Corvalan C, Araya M, Casanello P, Kusanovic JP, Uauy R. Effectiveness of a normative nutrition intervention (diet, physical activity and breastfeeding) on maternal nutrition and offspring growth: The Chilean maternal and infant nutrition cohort study (CHiMINCs). BMC Pregnancy Childbirth. 2015; 15(1):175. http://dx.doi.org/10.1 186/s12884-015-0605-1

20. Majrooh MA, Hasnain S, Akram J, Siddiqui A, Memon ZA. Coverage and Quality of antenatal care provided at primary health care facilities in the 
"punjab" province of "Pakistan". PLoS One. 2014; 9(11):e113390. http://dx.plos.org/10.1371/journal. pone.0113390

21. Fudação Sistema Estadual de Análise de Dados. Sistema de informações dos municípios paulistas. São Paulo: Seade; 2012 [acesso 2014 jun 11]. Disponível em: http://produtos.seade.gov.br/produtos/ perfil/perfilMunEstado.php

22. Instituto Brasileiro de Geografia e Estatística. IBGE cidades - Santos Censo. Brasília: IBGE; 2010 [acesso 2012 mar 25]. Disponível em: http://www.ibge. gov.br/cidadesat/link. php?codmun=354850

23. Brasil. Ministério da Saúde. Secretaria de Atenção à Saúde. Sistema de Informação da Atenção Básica - SIAB: atendimento pré-natal segundo município. Brasília: Ministério da Saúde; 2013.

24. Donabedian A. The quality of care: How can it be assessed? JAMA. 1988; 260(12):1743-8.

25. Dhalia CBC, Diaz-Bermudez, XP. Teste rápido: por que não? Estudos que contribuíram para a política de ampliação da testagem para o HIV no Brasil. Brasília: Ministério da Saúde; 2007.

26. Brasil. Ministério da Saúde. Secretaria de Atenção à Saúde. Saúde da criança: nutrição infantil: aleitamento materno e alimentação complementar; Cadernos de Atenção Básica, n 23. Brasília: Ministério da Saúde; 2009.

27. Elias PE, Ferreira CW, Alves MCG, Cohn A, Kishima V, Escrivão Junior $A$, et al. Atenção básica em saúde: comparação entre PSF e UBS por estrato de exclusão social no município de São Paulo. Ciênc Saúde Colet. 2006; 11(3):633-41.

28. Brasil. Ministério da Saúde. Secretaria de Atenção à Saúde. Manual de estrutura física das unidades básicas de saúde: saúde da família. $2^{a}$ ed. Brasília: Ministério da Saúde; 2008 [acesso 2013 out 5]. Disponível em: http://bvsms.saude.gov.br/bvs/ publicacoes/manual_estrutura_fisica_ubs.pdf

29. Brasil. Ministério da Saúde. Secretaria de Atenção à Saúde. Política Nacional de Atenção Básica. Brasília: Ministério da Saúde; 2007 [acesso 2011 out 21]. Disponível em: http://portal.saude.gov.br/ portal/arquivos/pdf/volume_4_completo.pdf

30. Camossa ACA, Telarolli Júnior R, Machado MLT. O fazer teórico-prático do nutricionista na estratégia saúde da família: representações sociais dos profissionais das equipes. Rev Nutr. 2012; 25(1):89-106. http://dx.doi.org/10.1590/S1415-52732012000 100009

31. Vettore MV, Dias M, Vettore MV, Leal MC. Avaliação da qualidade da atenção pré-natal dentre gestantes com e sem história de prematuridade no Sistema único de Saúde no Rio de Janeiro, Brasil. Rev Bras
Saúde Mater Infant. 2013; 13(2):89-100. http://dx. doi.org/10.1590/S1519-38292013000200002

32. Anversa ETR, Bastos GAN, Nunes LN, Dal Pizzol TS. Qualidade do processo da assistência pré-natal: unidades básicas de saúde e unidades de Estratégia Saúde da Família em município no Sul do Brasil. Cad Saúde Pública. 2012; 28(4):789-800. http:// dx.doi.org/10.1590/S0102-311X2012000400018

33. Tarekegn SM, Lieberman LS, Giedraitis V. Determinants of maternal health service utilization in Ethiopia: Analysis of the 2011 Ethiopian Demographic and Health Survey. BMC Pregnancy Childbirth. 2014; 14(1):161. http://dx.doi.org/ 10.1 186/1471-2393-14-161

34. Silva EP, Lima RT, Ferreira NLS, Costa MJDCE. Prénatal na atenção primária do município de João Pessoa-PB: caracterização de serviços e usuárias. Rev Bras Saúde Matern Infant. 2013; 13(1):29-37. http://dx.doi.org/10.1590/S15-19-38292013000/0 0004

35. Castro PDS, Castro MBT, Kac G. Aderência às recomendações dietéticas do Institute of Medicine (Estados Unidos) e o seu efeito no peso durante a gestação. Cad Saúde Pública. 2013; 29(7):1311-21. http://dx.doi.org/10.1590/S0102-311X2013000 700006

36. Qiaozhen L, Xiaoyang Z, Mclntosh T, Davis $H$, Nemeth JF, Pendley C, et al. Development of different analysis platforms with LC-MS for pharmacokinetic studies of protein drugs. Anal Chem. 2009; 81(21):8715-23. http://dx.doi.org/ 10.1021/ac901991x

37. Rasmussen V. Weight gain in pregnancy. Human reproduction. 1997 [cited 2012 Nov 14]. Available from: http://humrep.oxfordjournals.org/content/ 12/suppl_1/110.short

38. Serruya SJ, Cecatti JG, Lago TG. O Programa de Humanização no Pré-natal e Nascimento do Ministério da Saúde no Brasil: resultados iniciais. Cad Saúde Pública. 2004; 20(5):1281-9. http://dx.doi. org/10.1590/S0102-311X2004000500022

39. Caldeira AP, Oliveira RM, Rodrigues OA. Qualidade da assistência materno-infantil em diferentes modelos de Atenção Primária. Ciênc Saúde Colet. 2010; 15(Supl. 2):3139-47.

40. De Oliveira MIC, Camacho LA, Tedstone AE. Extending breastfeeding duration through primary care: A systematic review of prenatal and postnatal interventions. J Hum Lactation. 2001; 17(4):326-43.

41. Oliveira MIC, Camacho LAB, Oliveira Souza IE. Promoção, proteção e apoio à amamentação na atenção primária à saúde no Estado do Rio de Janeiro, 
Brasil: uma política. Cad Saúde Pública. 2005; 21(6):1901-10. http://dx.doi.org/10.1590/S0102-3 $11 \times 2005000600040$

42. Giugliani ERJ. O aleitamento materno na prática clínica. J Pediatr. 2000; 76(3):238-52.

43. Parada CMGDL, Carvalhaes MADBL, Winckler CC, Winckler LA, Winckler VC. Situação do aleitamento materno em população assistida pelo programa de saúde da família-PSF. Rev Latino-Am Enfermagem. 2005; 13(3):407-14.

44. Pádua JG, Boog MCF. Avaliação da inserção do nutricionista na Rede Básica de Saúde dos muni- cípios da Região Metropolitana de Campinas. Rev Nutr. 2006; 19(4):413-24. http://dx.doi.org/10.15 90/S1415-52732006000400001

45. Brasil. Conselho Federal de Nutricionistas. O papel do nutricionista na atenção primária à saúde. Brasília: Conselho Federal de Nutricionistas; 2008 [acesso 2013 jun 15]. Disponível em: http://www. cfn.org. br/eficiente/repositorio/cartilhas/61.pdf

Recebido: novembro 11, 2014 Versão final: agosto 8, 2015 Aprovado: setembro 22, 2015 
